Difan Tang, Lei Chen, Zhao Feng Tian, Eric Hu

Developing a virtual stiffness-damping system for airfoil aeroelasticity testing Proceedings of the 20th IEEE International Conference on Industrial Technology (ICIT 2019), 2019 / vol.2019-February, pp.96-101

\title{
(C2019 IEEE.
}

Published version at: http://dx.doi.org/10.1109/ICIT.2019.8755200

\section{PERMISSIONS}

\section{https://www.ieee.org/publications/rights/author-posting-policy.html}

\section{Author Posting of IEEE Copyrighted Papers Online}

The IEEE Publication Services \& Products Board (PSPB) last revised its Operations Manual Section 8.1.9 on Electronic Information Dissemination (known familiarly as "author posting policy") on 7 December 2012.

PSPB accepted the recommendations of an ad hoc committee, which reviewed the policy that had previously been revised in November 2010. The highlights of the current policy are as follows:

- The policy reaffirms the principle that authors are free to post their own version of their IEEE periodical or conference articles on their personal Web sites, those of their employers, or their funding agencies for the purpose of meeting public availability requirements prescribed by their funding agencies. Authors may post their version of an article as accepted for publication in an IEEE periodical or conference proceedings. Posting of the final PDF, as published by IEEE Xplore ${ }^{\circledR}$, continues to be prohibited, except for open-access journal articles supported by payment of an article processing charge (APC), whose authors may freely post the final version.

- $\quad$ The policy provides that IEEE periodicals will make available to each author a preprint version of that person's article that includes the Digital Object Identifier, IEEE's copyright notice, and a notice showing the article has been accepted for publication.

- $\quad$ The policy states that authors are allowed to post versions of their articles on approved third-party servers that are operated by not-for-profit organizations. Because IEEE policy provides that authors are free to follow public access mandates of government funding agencies, IEEE authors may follow requirements to deposit their accepted manuscripts in those government repositories.

IEEE distributes accepted versions of journal articles for author posting through the Author Gateway, now used by all journals produced by IEEE Publishing Operations. (Some journals use services from external vendors, and these journals are encouraged to adopt similar services for the convenience of authors.) Authors' versions distributed through the Author Gateway include a live link to articles in IEEE Xplore. Most conferences do not use the Author Gateway; authors of conference articles should feel free to post their own version of their articles as accepted for publication by an IEEE conference, with the addition of a copyright notice and a Digital Object Identifier to the version of record in IEEE Xplore.

\section{June 2020}




\section{Developing a Virtual Stiffness-Damping System for Airfoil Aeroelasticity Testing}

\author{
$1^{\text {st }}$ Difan Tang \\ School of Mechanical Engineering \\ The University of Adelaide \\ Adelaide, Australia \\ difan.tang@adelaide.edu.au
}

\author{
$2^{\text {nd }}$ Lei Chen \\ School of Mechanical Engineering \\ The University of Adelaide \\ Adelaide, Australia \\ lei.chen@ adelaide.edu.au
}

\author{
$3^{\text {rd }}$ Zhao Feng Tian \\ School of Mechanical Engineering \\ The University of Adelaide \\ Adelaide, Australia \\ zhao.tian@adelaide.edu.au
}

\author{
$4^{\text {th }}$ Eric $\mathrm{Hu}$ \\ School of Mechanical Engineering \\ The University of Adelaide \\ Adelaide, Australia \\ eric.hu@adelaide.edu.au
}

\begin{abstract}
Development of a two-degrees-of-freedom (2-DOF) virtual stiffness-damping system (VSDS) to facilitate industrial and laboratory testing of airfoil aeroelastic instability (AAT) is presented in this paper. Development and use of existing testbeds are costly due to involvement of physical springs to simulate airfoil elasticity. Although replacing physical springs with a VSDS has been used in other fields such as marine and biomechanics engineering, existing VSDSs cannot be directly used for AAT due to operation requirements and conditions being different. Therefore, in this study a new VSDS is developed specifically for AAT. Firstly, the concept of 1-DOF VSDS is extended to 2 DOFs, with the dynamics coupling between each DOF addressed at the stage of operation principle determination, by proposing direct force/torque regulation with force/torque feedback. Secondly, resolution loss in velocity measurement is identified as a main problem associated with the non-reduction transmission required and is solved by proposing a modified extended-state observer (MESO) for fast velocity estimation. Thirdly, system identification and calibration procedures involved in developing the new VSDS is reduced to minimum by applying a robust force/torque tracking controller. As validated in wind-tunnel experiments the new VSDS can closely track the desired force/torque and provide satisfactory virtual stiffness and damping in AAT.

Index Terms-aeroelasticity, virtual stiffness, virtual damping
\end{abstract}

\section{INTRODUCTION}

Aeroelasticity studies the interaction between aerodynamic loads and elastic bodies. Airfoil flutter, as typical dynamic aeroelastic instability, can cause airfoil structure fatigue and failure. For research on airfoil flutter and suppression, numerous facilities have been built [1]-[4]. Development and use of these test-beds can be costly and time-consuming when a wide range of stiffness needs to be investigated, requiring different materials or structures for scaled prototypes [2], [4] and different physical springs for typical airfoil sections [1], [3]. A more efficient and low-cost alternative arose in marine engineering, using electric drives to simulate springs and dampers [5]-[10].

This work has been supported by an Australian Government Research Training Program Scholarship and the Sir Ross \& Sir Keith Smith Fund.
Some other related studies can also be seen in biomechanics applications, with corresponding purposes, requirements, and operation conditions being much different [11]. For unified naming, these approaches are termed as 'virtual stiffnessdamping systems' (VSDSs). To apply the VSDS concept in airfoil aeroelasticity testing (AAT), a new VSDS is developed in this study specifically for AAT, for the first time, filling the gaps with the following contributions:

1) The existing VSDSs are of one degree-of-freedom (DOF) and no VSDS is available for AAT where at least two DOFs are required. In particular, the dynamics coupling between each DOF is addressed in this study;

2) Reduction transmission is commonly used in the existing VSDSs but not preferred for AAT where easy back-driving by aerodynamic loads is desired as one of the flutter triggering conditions. As a first trial on VSDS, this study uses non-reduction transmission and solves associated problems.

3) On the existing VSDSs, transmission power-loss is generally modeled and estimated from offline system identification procedures, and can be inaccurate in the presence of uncertainties and parameter change over time or under different operation conditions. In the interests of enhanced robustness and ease of maintenance with simplified system identification and calibration procedures, online power-loss estimation and compensation is proposed.

\section{Aeroelastic Model}

The aeroelastic system under consideration can be fundamentally described by a two-degrees-of-freedom (2-DOF) model (Fig. 1) for subsonic regime, which assumes a rigid airfoil supported by elastic elements [3] with corresponding equation of motion being

$$
\boldsymbol{m} \ddot{\boldsymbol{q}}+\boldsymbol{c} \dot{\boldsymbol{q}}+\boldsymbol{k} \boldsymbol{q}+\boldsymbol{F}_{c}=\boldsymbol{F}_{\text {aero }},
$$

with

$$
\boldsymbol{q}=\left[\begin{array}{c}
h \\
\alpha
\end{array}\right], \quad \boldsymbol{F}_{\text {aero }}=\left[\begin{array}{c}
-F_{l} \\
F_{m}
\end{array}\right], \quad \boldsymbol{F}_{c}=\left[\begin{array}{c}
-m_{w} \dot{\alpha}^{2} r_{\alpha} b \sin \alpha \\
0
\end{array}\right],
$$




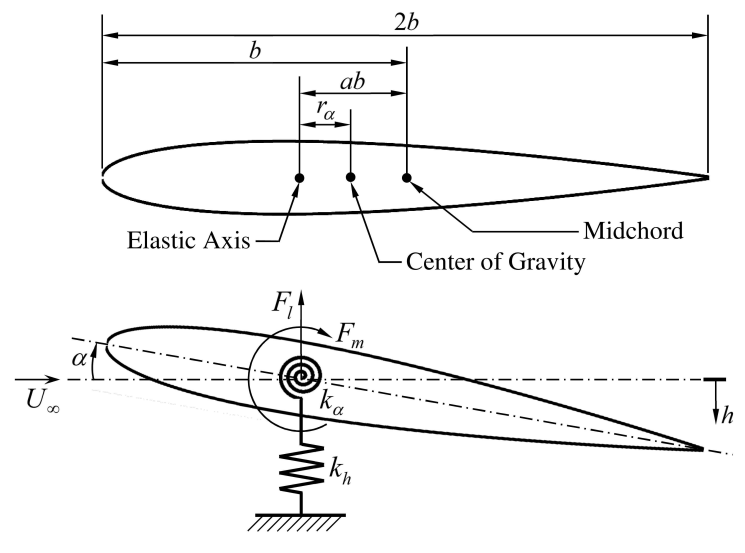

Fig. 1. Schematic diagram of a 2-DOF aeroelastic system [3].

$$
\begin{gathered}
\boldsymbol{m}=\left[\begin{array}{cc}
m_{w} & m_{w} r_{\alpha} b \cos \alpha \\
m_{w} r_{\alpha} b \cos \alpha & I_{\alpha}
\end{array}\right] \\
\boldsymbol{c}=\left[\begin{array}{cc}
c_{h} & 0 \\
0 & c_{\alpha}
\end{array}\right], \quad \boldsymbol{k}=\left[\begin{array}{cc}
k_{h} & 0 \\
0 & k_{\alpha}
\end{array}\right] \\
F_{l}=\rho U_{\infty}^{2} b^{2} l_{s} C_{l \alpha}\left[\alpha+\dot{h} / U_{\infty}+(0.5-a) b \dot{\alpha} / U_{\infty}\right] \\
F_{m}=\rho U_{\infty}^{2} b^{3} l_{s} C_{m_{\alpha e}}\left[\alpha+\dot{h} / U_{\infty}+(0.5-a) b \dot{\alpha} / U_{\infty}\right] \\
C_{l \alpha}=\partial C_{l} / \partial \alpha, \quad C_{m \alpha}=\partial C_{m} / \partial \alpha \\
C_{m_{\alpha e}}=(0.5+a) C_{l \alpha}+2 C_{m \alpha}
\end{gathered}
$$

where geometry and force related parameters and variables are defined in Fig. 1, and other terms are defined as

$m_{w}: \quad$ airfoil mass;

$I_{\alpha}$ : airfoil rotational inertia about its elastic axis;

$k_{h}, k_{\alpha}$ : Stiffness coefficients of plunge and pitch DOFs;

$c_{h}, c_{\alpha}$ : Damping coefficients of plunge and pitch DOFs;

$\rho: \quad$ air density;

$l_{s}: \quad$ airfoil span;

$C_{l}$ : airfoil lift coefficient;

$C_{m}$ : airfoil moment coefficient at $1 / 4$ chord;

$U_{\infty}$ : airflow velocity.

Denote the forces resulted from structural stiffness and damping by $\boldsymbol{F}_{s}$. Then according to (1), there is

$$
\boldsymbol{F}_{s}(\boldsymbol{q}, \dot{\boldsymbol{q}})=\boldsymbol{c} \dot{\boldsymbol{q}}+\boldsymbol{k} \boldsymbol{q} .
$$

In the absence of physical springs, $\boldsymbol{F}_{s}(\boldsymbol{q}, \dot{\boldsymbol{q}})$ can be generated by a virtual stiffness-damping system instead.

\section{VirTuAl StiffNeSS-DAMPING System}

\section{A. Operation Principle Determination}

The virtual stiffness-damping system (VSDS) for airfoil aeroelasticity testing (AAT) needs at least two degrees-offreedom (DOFs), and the primary concern is the dynamics coupling between each DOF, as mentioned in Section I.

Equation (1) shows the two DOFs are coupled in plunge and pitch displacements. If virtual stiffness and damping are realized via position control with force feedback [5]-[7], realtime calculation of the reference position needs to consider the inertial and aerodynamic coupling. Since (1) is a secondorder approximation of general nonlinear aeroelastic systems, modeling errors can cause inaccuracy of simulated stiffness and damping under position control.

It can also be seen from (1) that the sums $c_{h} \dot{h}+k_{h} h$ and $c_{\alpha} \dot{\alpha}+k_{\alpha} \alpha$, each considered as a single variable, are mutually independent between the two DOFs. If the stiffness and damping of the airfoil structure are physically simulated by taking direct control on corresponding force/torque generation according to the two independent sums, then subsequent mechanical design and control synthesis will not be affected by the dynamics coupling in plunge displacement and pitch angle. In this regard, the computed force/torque control without the need for force/torque feedback as in [8]-[10] fits.

However, to meet the requirements on enhanced robustness and ease of maintenance with simplified system identification and calibration procedures, it is desired to close the control loop with force/torque feedback. This drives the VSDS operation principle into a new direction - direct force/torque regulation with force/torque feedback.

\section{B. Mechanical Design}

Following the operation principle proposed in Section III-A, a new VSDS is developed in this study. The overview of the computer-aided-design (CAD) model and structural details are given in Figs. 2 and 3, respectively.

To allow back-driving with least resistance so that the plunge DOF can be easily perturbed by aerodynamic loads, speed amplification instead of reduction is introduced via two pairs of synchronous-belt transmission (Fig. 3(a)). Similarly, the pitch-DOF motor is directly connected to the pitching shaft (elastic axis) of the airfoil without any speed reduction mechanism (Fig. 3(b)). As discussed in Section I, this transmission configuration is a first trial for VSDS in AAT and potential problems need to be addressed (to be covered in Section III-C).

\section{Velocity Measurement and Estimation}

It has been known that velocity measurement using encoders suffers from precision loss at low velocities crossing zero, which is worse for low-resolution encoders [12]-[14]. In the case of the proposed VSDS, the non-reduction transmission on the other hand also results in some extent of precision loss despite high-resolution encoders used. This leads to imprecise measurement of low velocities. Remedies given in [12]-[14], however, are not ideal solutions to the proposed VSDS due to the dynamics coupling in plunge/pitch displacements. Motivated by the concept of the extended-state observer (ESO) [15] seen in applications with strong nonlinearities [16]-[18], we derived a modified ESO (MESO) for velocity estimation. Let $\boldsymbol{z}_{1}=\boldsymbol{q}, \boldsymbol{z}_{2}=\dot{\boldsymbol{q}}_{1}$. Then (1) has a more general form

$$
\left\{\begin{array}{l}
\dot{z}_{1}=\boldsymbol{z}_{2}, \\
\dot{\boldsymbol{z}}_{2}=\boldsymbol{f}\left(\boldsymbol{z}_{1}, \boldsymbol{z}_{2}\right),
\end{array}\right.
$$




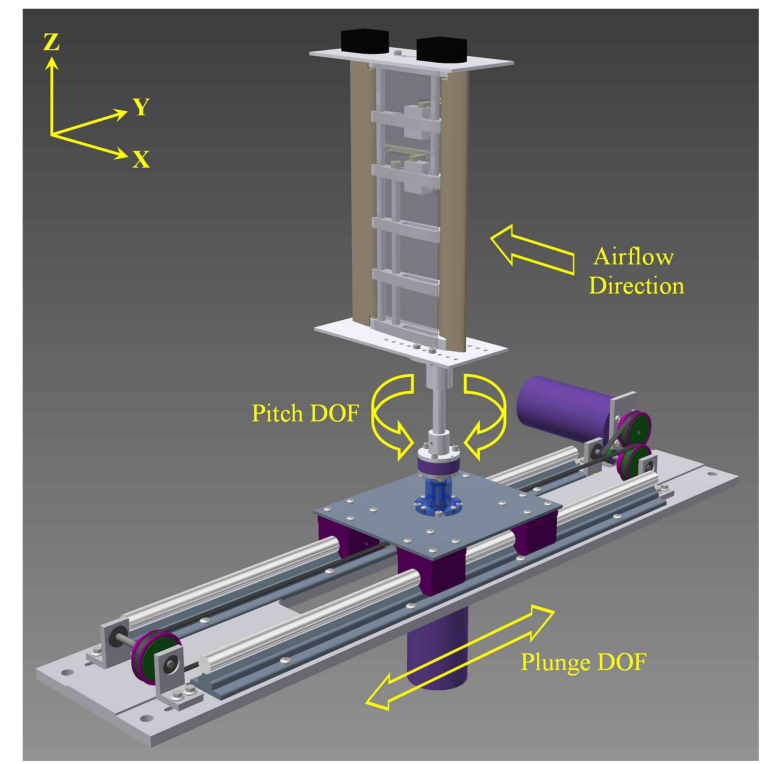

Fig. 2. Overview of the CAD modeling of the new VSDS prototype.

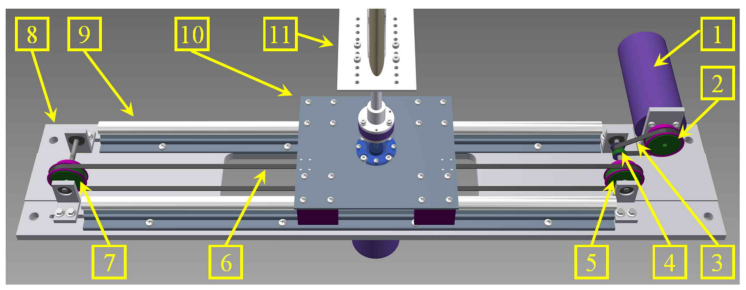

(a) Plunge DOF (perspective top-front view)

1, plunge-DOF motor

2 , pulley P1 (44 grooves)

3 , synchronous belt BI

4 , pulley P2 (14 grooves)

5 , pulley P3 (44 grooves)

6 , synchronous belt B2

7, pulley $\mathrm{P} 4$ (44 grooves)

8 , main platform

9 , linear rail

10 , sliding carriage

11, airfoil

12 , pitching shaft (airfoil)

13, force/torque transducer

14 , shaft housing

15 , pitching shaft (VSDS)

16 , coupler

17, pitch-DOF motor

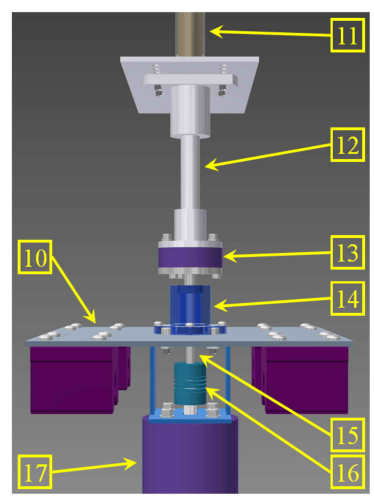

(b) Pitch DOF

(perspective front view)

Fig. 3. Structural details of the new VSDS prototype.

where $\boldsymbol{f}\left(\boldsymbol{z}_{1}, \boldsymbol{z}_{2}\right)=\boldsymbol{m}^{-1}\left(\boldsymbol{F}_{\text {aero }}-\boldsymbol{F}_{c}-\boldsymbol{c} \dot{\boldsymbol{q}}-\boldsymbol{k} \boldsymbol{q}\right)+\boldsymbol{\zeta}\left(t, \boldsymbol{z}_{1}, \boldsymbol{z}_{2}\right)$, with $\boldsymbol{\zeta}\left(t, \boldsymbol{z}_{1}, \boldsymbol{z}_{2}\right)$ containing un-modeled dynamics.

Let $\hat{z}_{1}, \hat{z}_{2}$, and $\hat{z}_{3}$ denote the estimate of $\boldsymbol{z}_{1}, \boldsymbol{z}_{2}$, and $\boldsymbol{f}\left(\boldsymbol{z}_{1}, \boldsymbol{z}_{2}\right)$, respectively, and $\boldsymbol{e}_{z}=\hat{\boldsymbol{z}}_{1}-\boldsymbol{q}$. The MESO for system (3) is constructed as

$$
\left\{\begin{array}{l}
\dot{\hat{\boldsymbol{z}}}_{1}=\hat{z}_{2}-p_{1} \boldsymbol{e}_{z} \\
\dot{\hat{z}}_{2}=\hat{z}_{3}-p_{2} \boldsymbol{e}_{z} / \sqrt{\boldsymbol{e}_{z}^{\mathrm{T}} \boldsymbol{e}_{z}+1} \\
\dot{\hat{\boldsymbol{z}}}_{3}=-p_{3} \boldsymbol{e}_{z} / \sqrt{\boldsymbol{e}_{z}^{\mathrm{T}} \boldsymbol{e}_{z}+1}
\end{array}\right.
$$

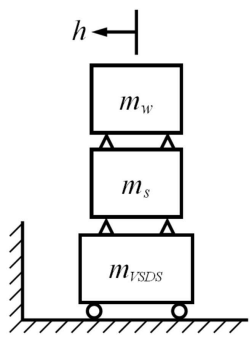

(a) Reference frame (b) Forces on isolated bodies

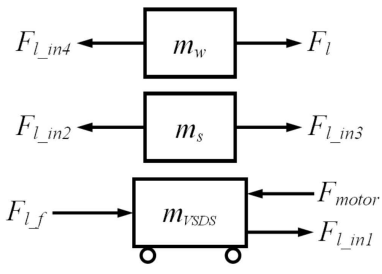

Fig. 4. The VSDS plunge DOF as a multi-body system.

where $p_{1} \in \mathbb{R}^{+}, p_{2} \in \mathbb{R}^{+}$and $p_{3} \in \mathbb{R}^{+}$are design parameters.

The MESO parameters can be initially selected by evaluating $\boldsymbol{e}_{z}$, and further adjusted by comparing $z_{2}$ with the estimation from a conventional approximation filter (to be demonstrated in Section IV).

\section{Force/Torque Measurement and Control}

For force/torque measurement, a 6-axis force/torque transducer (ATI ${ }^{\circledR}$ Mini40) is mounted between the pitching shafts of the VSDS and the airfoil. Under this arrangement, the airfoil and its pitching shaft together are considered as one whole rigid body, the total mass of which is $m_{w}$ according to (1).

With the force/torque transducer regarded as an independent rigid body, the plunge DOF can be considered as a multi-body system illustrated in Fig. 4(a), which has the same reference frame as Fig. 1. Corresponding free-body diagram of the isolated multiple bodies is given in Fig. 4(b). Accordingly,

$$
\begin{gathered}
m_{w} \ddot{h}+F_{l \_i n 4}=F_{l}, \\
m_{s} \ddot{h}+F_{l \_i n 2}=F_{l \_i n 3}, \\
m_{\text {VSDs }} \ddot{h}+F_{l_{\_} f}+F_{l \_i n 1}=F_{m o t o r}, \\
F_{l \_i n 1}=F_{l \_i n 2}, \\
F_{l_{-} i n 3}=F_{l \_i n 4},
\end{gathered}
$$

where $F_{\text {motor }}$ is the translational force (tension) on the synchronous belt B1; $F_{l_{-} f}$ is the total equivalent friction along the entire plunge-DOF power transmission from motor output to the sliding carriage, consisting of viscous, Coulomb and Stribeck frictions; $F_{l_{-} i n 1}, F_{l_{-} i n 2}, F_{l_{-} i n 3}$ and $F_{l_{-} i n 4}$ are internal forces defined according to Newton's third law of motion; $m_{s}$ is the mass of the force/torque transducer, and $m_{\text {VSDS }}$ is the equivalent mass of the plunge DOF transmission.

Let $\hat{c}_{h}, \hat{k}_{h}, \hat{c}_{\alpha}$, and $\hat{k}_{\alpha}$ denote the coefficients of simulated virtual stiffness and damping of respective DOFs. Comparing (1) and (5) gives

$$
F_{l \_i n 4}=-\hat{c}_{h} \dot{h}-\hat{k}_{h} h .
$$

Similarly, for the pitch DOF we have

$$
F_{m_{-} i n 4}=\hat{c}_{\alpha} \dot{\alpha}+\hat{k}_{\alpha} \alpha,
$$

where $F_{m_{-} i n 4}$ is the internal torque that contributes to

$$
I_{\alpha} \ddot{\alpha}+F_{m \_i n 4}=F_{m} \text {. }
$$




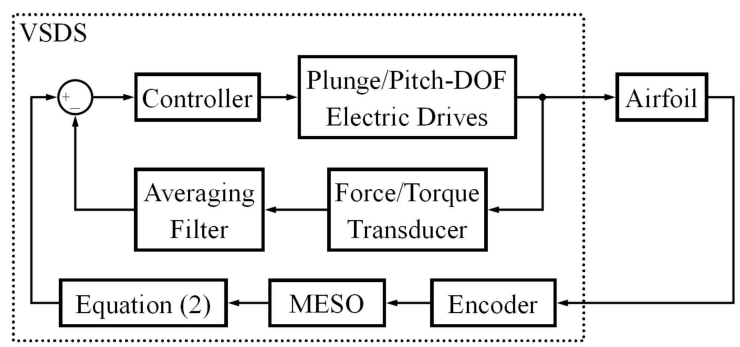

Fig. 5. Schematic diagram of the proposed VSDS control system.

TABLE I

EXPERIMENTALLY IDENTIFIED DYNAMICS OF THE VSDS PROTOTYPE

\begin{tabular}{cccccc}
\hline \multirow{2}{*}{ DOF } & \multicolumn{4}{c}{ Matrices of System Dynamics } & \multirow{2}{*}{ Fit (\%) } \\
\cline { 2 - 5 } Plunge & {$\left[\begin{array}{ll}-8.791 & \multicolumn{2}{c}{\boldsymbol{A}} & \boldsymbol{B} & \boldsymbol{C} & \boldsymbol{D} & \\
-273.8 & -165.9\end{array}\right]$} & {$\left[\begin{array}{c}45.5 \\
29.19\end{array}\right]$} & {$\left[\begin{array}{c}25.53 \\
3.903\end{array}\right]^{\mathrm{T}}$} & 0 & 93.88 \\
Pitch & {$\left[\begin{array}{cc}18.67 & -464 \\
429.1 & -716.3\end{array}\right]$} & {$\left[\begin{array}{c}5823 \\
7501\end{array}\right]$} & {$\left[\begin{array}{c}0.4412 \\
-0.0669\end{array}\right]^{\mathrm{T}}$} & 0 & 94.04 \\
\hline \hline
\end{tabular}

Let $\hat{\boldsymbol{F}}_{s} \triangleq\left[\begin{array}{ll}-F_{l} \_i n 4 & F_{m \_i n 4}\end{array}\right]^{\mathrm{T}}$. Then writing (10) and (11) in matrix form yields

$$
\hat{\boldsymbol{F}}_{s}(\boldsymbol{q}, \dot{\boldsymbol{q}})=\hat{\boldsymbol{c}} \dot{\boldsymbol{q}}+\hat{\boldsymbol{k}} \boldsymbol{q},
$$

where $\hat{\boldsymbol{c}}=\operatorname{diag}\left(\hat{c}_{h}, \hat{c}_{\alpha}\right)$ and $\hat{\boldsymbol{k}}=\operatorname{diag}\left(\hat{k}_{h}, \hat{k}_{\alpha}\right)$.

According to the force/torque transducer structure as well as (5) and (12), force/torque measurements closely take the value of $\hat{\boldsymbol{F}}_{s}$. The desired $\boldsymbol{k}$ and $\boldsymbol{c}$ can be approximated by proper control of the motor torque so that $\hat{\boldsymbol{F}}_{s} \rightarrow \boldsymbol{F}_{s}$, according to (7). This requires correct knowledge and proper compensation of power loss caused by frictions as well as other un-modeled dynamics and exogenous disturbances. Without force/torque feedback, extensive system identification procedures are necessary [8]-[10]. To meet requirements on enhanced robustness and ease of maintenance with simplified system identification and calibration procedures, the total power loss can be dynamically estimated online by evaluating $\hat{\boldsymbol{F}}_{s}$ against reference $\boldsymbol{F}_{s}$, and the VSDS control system is proposed as in Fig. 5.

To assist VSDS controller design, dynamics of both DOFs of the VSDS prototype were obtained via black-box system identification with voltages as inputs and force/torque measurement as outputs. Chirp signals of 2 to $5 \mathrm{~Hz}$ were used to explore up to $5 \%$ and $60 \%$ rated capacity (sufficient for required force/torque) of pitch- and plunge-DOF motors, respectively. Estimated models (Table. I) are in state-space linear time-invariant form as

$$
\left\{\begin{array}{l}
\dot{x}=A x+B u, \\
y=C x+D u,
\end{array}\right.
$$

where vectors $\boldsymbol{x}, \boldsymbol{y}$, and $\boldsymbol{u}$ contain states, outputs, and inputs, respectively; $\boldsymbol{A}, \boldsymbol{B}, \boldsymbol{C}$, and $\boldsymbol{D}$ are system matrices.

Power loss is partly captured in the system identification, and the identified models of the best fit are selected. Although modeling errors and other types of disturbances (together

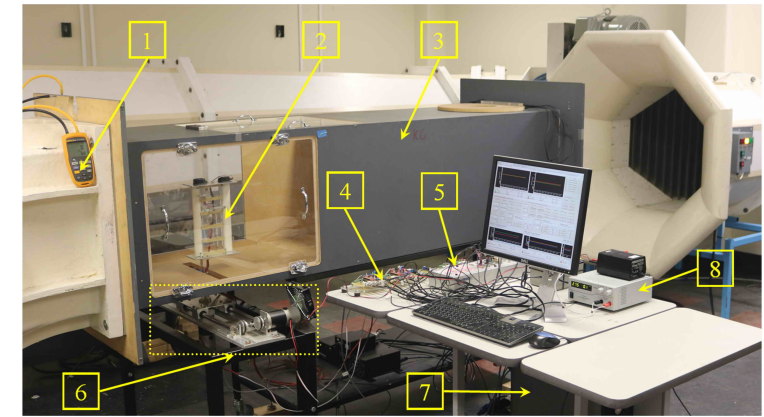

1 - pressure transducer connected to a Pitot tube; 2 - airfoil; 3 - wind tunnel test duct; 4 - I/O board; 5 - dSPACE ${ }^{\mathbb{R}}$ DS1104 R\&D board; 6 - VSDS; 7 - PC; 8 - power.

Fig. 6. Wind-tunnel experiment setup.

defined as 'unknown inputs') inevitably exist, corresponding impacts on the accuracy of virtual stiffness and damping simulated by electric drives can be minimized by applying the linear-quadratic-Gaussian (LQG) tracking control integrated with an unknown-input estimator (UIE) [19]. Detailed composition of the controller, without distinguishing between specific DOFs, is given below in a general multi-input multi-output form.

The control $\boldsymbol{u}$ is given by $\boldsymbol{u}=\boldsymbol{u}_{c}-\hat{\boldsymbol{d}}_{e}$, where $\boldsymbol{u}_{c}$ comes from the LQG component for trajectory tracking, and $\hat{\boldsymbol{d}}_{e}$ is the estimated equivalent total power loss due to unknown inputs.

The nominal control $\boldsymbol{u}_{c}$ takes the form of

$$
\boldsymbol{u}_{c}=-\boldsymbol{K}_{x} \hat{\boldsymbol{x}}-\boldsymbol{K}_{w} \boldsymbol{x}_{w}+\boldsymbol{K}_{r} \boldsymbol{F}_{s}(\boldsymbol{q}, \dot{\boldsymbol{q}}),
$$

with $\dot{\boldsymbol{x}}_{w}=\boldsymbol{F}_{s}(\boldsymbol{q}, \dot{\boldsymbol{q}})-\boldsymbol{y}=\boldsymbol{F}_{s}(\boldsymbol{q}, \dot{\boldsymbol{q}})-\boldsymbol{C} \boldsymbol{x}$, where $\boldsymbol{K}_{x}, \boldsymbol{K}_{w}$, and $\boldsymbol{K}_{r}$ are LQG gains and can be selected following standard LQG design procedures.

Estimated power loss $\hat{\boldsymbol{d}}_{e}$ is obtained through the UIE as

$$
\hat{\boldsymbol{d}}_{e}=\hat{\boldsymbol{d}}_{e v}+\boldsymbol{K}_{d}(\boldsymbol{y}-\hat{\boldsymbol{y}}),
$$

with

$$
\left\{\begin{array} { l } 
{ \dot { \boldsymbol { x } } _ { f } = \boldsymbol { A } _ { f } \boldsymbol { x } _ { f } + \boldsymbol { B } _ { f } \hat { \boldsymbol { d } } _ { e } , } \\
{ \hat { \boldsymbol { d } } _ { e v } = \boldsymbol { C } _ { f } \boldsymbol { x } _ { f } , }
\end{array} \quad \left\{\begin{array}{l}
\dot{\hat{\boldsymbol{x}}}=\boldsymbol{A} \hat{\boldsymbol{x}}+\boldsymbol{B} \boldsymbol{u}_{c}+\boldsymbol{L}(\boldsymbol{y}-\hat{\boldsymbol{y}}), \\
\hat{\boldsymbol{y}}=\boldsymbol{C} \hat{\boldsymbol{x}}
\end{array}\right.\right.
$$

where $\boldsymbol{K}_{d}$ is the UIE gain; $\hat{\boldsymbol{y}}$ contains estimated force/torque; $\boldsymbol{A}_{f}, \boldsymbol{B}_{f}$, and $\boldsymbol{C}_{f}$ are matrices of a low-pass-filter-characterized subsystem denoted as $\boldsymbol{G}_{f}(s)$ in frequency domain; $\boldsymbol{L}$ is the state observer gain; $\hat{\boldsymbol{x}}$ contains estimated states.

The UIE gain $\boldsymbol{K}_{d}$ can be designed via linear-quadratic optimization, which finds gain $\boldsymbol{K}_{v}$ for the observable pair $(\boldsymbol{A}-\boldsymbol{L} \boldsymbol{C}, \boldsymbol{C})$ and yields $\boldsymbol{K}_{d}=\boldsymbol{B}^{\dagger} \boldsymbol{K}_{v}^{\mathrm{T}}$ with $\boldsymbol{B}^{\dagger}$ being the Moore-Penrose pseudo inverse of $\boldsymbol{B}$.

\section{EXPERIMENTAL VALIDATION}

To validate the new VSDS in providing virtual stiffness and damping in airfoil aeroelasticity testing (AAT), wind-tunnel experiments were conducted. The setup is shown in Fig. 6, with parameters listed in Table. II.

Two test scenarios are presented in this paper with corresponding settings listed in Table. III. In each scenario, comparisons were drawn between standard LQG tracking control and 
TABLE II

PARAMETERS OF Wind-TUNNEL EXPERIMENT SETUP

\begin{tabular}{cccc}
\hline \hline Parameters & Values & Parameters & Values \\
\hline$a$ & -0.569 & $c_{h}, c_{\alpha}, k_{h}, k_{\alpha}$ & See Table. III \\
$b$ & $0.075 \mathrm{~m}$ & $m_{\alpha}$ & $0.851 \mathrm{~kg}$ \\
$l_{s}$ & $0.260 \mathrm{~m}$ & $I_{\alpha}$ & $2.431 \times 10^{-3} \mathrm{~kg} \cdot \mathrm{m}^{2}$ \\
$r_{\alpha}$ & $0.033 \mathrm{~m}$ & $C_{l \alpha}$ & 6.573 \\
$\rho$ & $1.225 \mathrm{~kg} / \mathrm{m}^{3}$ & $C_{m \alpha}$ & 0 \\
\hline \hline
\end{tabular}

TABLE III

WIND-TUNNEL EXPERIMENT SCENARIOS

\begin{tabular}{|c|c|c|c|}
\hline Case & Flutter Boundary & Airflow Speed & Stiffness \& Damping \\
\hline 1 & $13.92 \mathrm{~m} / \mathrm{s}$ & $14.8 \mathrm{~m} / \mathrm{s}$ & $\begin{array}{l}k_{h}=50+300 h^{2} \mathrm{~N} / \mathrm{m} \\
k_{\alpha}=0.3+30 \alpha^{2} \mathrm{Nm} / \mathrm{rad} \\
c_{h}=14 \mathrm{~kg} / \mathrm{s} \\
c_{\alpha}=0.042 \mathrm{~kg} \cdot \mathrm{m}^{2} / \mathrm{s}\end{array}$ \\
\hline 2 & $16.02 \mathrm{~m} / \mathrm{s}$ & $16.8 \mathrm{~m} / \mathrm{s}$ & $\begin{array}{l}k_{h}, k_{\alpha}, c_{h}: \text { same as Test } 1 \\
k_{\alpha}=0.77+30 \alpha^{2} \mathrm{Nm} / \mathrm{rad}\end{array}$ \\
\hline
\end{tabular}

TABLE IV

VSDS CONTROLLER PARAMETERS

\begin{tabular}{ccccccc}
\hline \hline \multicolumn{2}{c}{$\boldsymbol{K}_{x}$} & $K_{w}$ & $K_{r}$ & $\boldsymbol{L}$ & $K_{d}$ & $G_{f}(s)$ \\
\hline$\left[\begin{array}{ll}6.27 & 3.98\end{array}\right]$ & -3.16 & 0.4 & {$\left[\begin{array}{ll}9.49 & 1.07\end{array}\right]^{\mathrm{T}}$} & 6.64 & $\frac{1}{(0.01 s+1)}$ \\
{$\left[\begin{array}{ll}4.39 & -0.74\end{array}\right]$} & -3.16 & 10.08 & {$\left[\begin{array}{ll}7.33 & 2.19\end{array}\right]^{\mathrm{T}}$} & 4.34 & $\frac{1}{(0.01 s+1)}$ \\
\hline \hline
\end{tabular}

the UIE-LQG control. The MESO was fined tuned to give fast estimation of velocities, with parameters $p_{1}=90, p_{2}=20000$, and $p_{3}=80000$. The UIE-LQG parameters are given in Table. IV. The phase lag introduced by the VSDS control system, from actual displacement to generated force/torque, was found to have a maximum of 4 degrees for flutter frequency of 2 to $3 \mathrm{~Hz}$ experienced in experiments.

Airfoil plunge and pitch responses in the two test scenarios are shown in Figs. 7 and 8. Proper velocity estimation by MESO can be verified by looking at both position and velocity estimation. To demonstrate, Fig. 9 shows a close-up of position/velocity MESO estimation for UIE-LQG control in Case 1, where conventional velocity approximation using a filter is also presented. Subject to the available resolution, the filter was designed as $\frac{s}{1 / 50 s+1}$ to provide acceptable velocity approximation, which however, introduced a large amount of phase lag compared with MESO estimation, as can be seen in Fig. 9. It can also be observed that position estimations using MESO closely follow encoder measurements, indicating fast and proper velocity estimation by MESO.

Note the different aeroelastic responses under the same experiment settings with different controllers, causes of which are revealed in Figs. 10 to 13. Significant differences between measurements and reference trajectories can be observed in tests with standard LQG control (Figs. 10 and 12). As a result, flutter failed to initiate under the LQG control (Figs. 7 and 8), although the airflow speeds in tests were higher than corresponding flutter boundaries. In comparison, the measured plunge-DOF forces and pitch-DOF torques strictly follow the
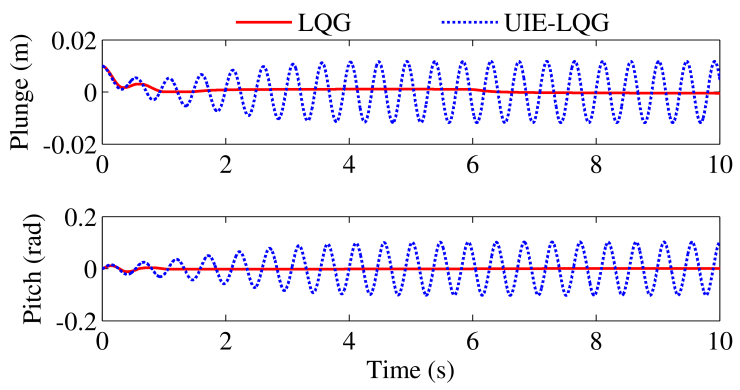

Fig. 7. Aeroelastic responses in Case 1 tests.
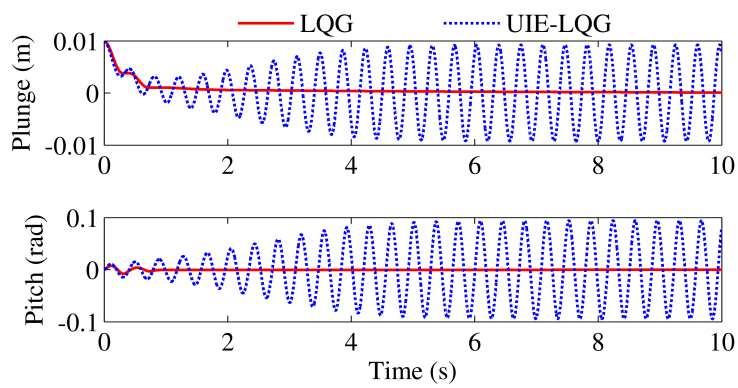

Fig. 8. Aeroelastic responses in Case 2 tests.

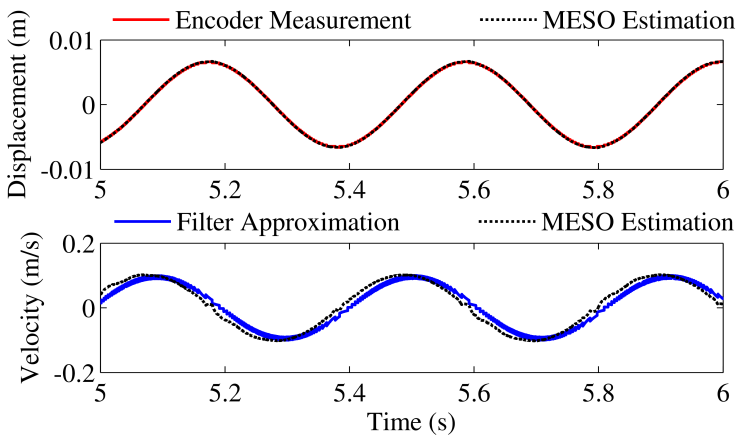

Fig. 9. Position/velocity estimation using MESO (Case 1, UIE-LQG).

desired trajectories under the UIE-LQG control, with tracking deviations barely identified (Figs. 11 and 13). This enabled successful initiation and development of flutter (Figs. 7 and 8).

Results show that: (1) Resolution loss in velocity measurements due to using non-reduction transmission can be solved by the proposed MESO; (2) Power loss due to unknown inputs, if not properly treated, have considerable impacts on VSDS performance; (3) Effective estimation and compensation of power loss requires enhanced controller robustness and the UIE-LQG control fits, with superior force/torque tracking achieved; (4) The maximum of 4 degrees displacement-toforce/torque phase lag has minor impacts on the overall simulation of physical stiffness and damping for AAT.

\section{CONClusion}

Motivated by the disadvantages of conventional test-beds for AAT and limitations of existing VSDSs in other fields, we develop a new VSDS for AAT in this study. The new 

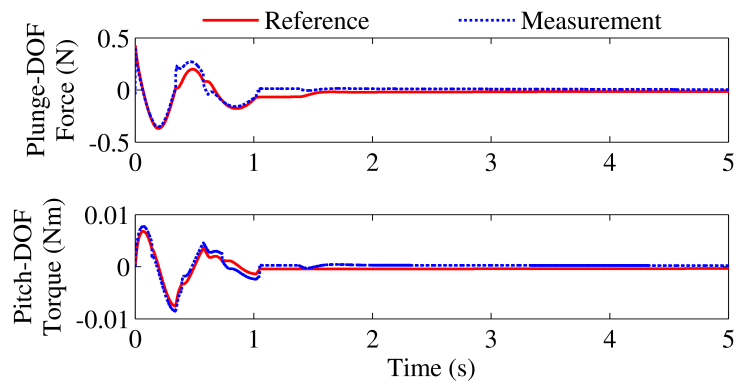

Fig. 10. VSDS force/torque under LQG control in Case 1 tests.
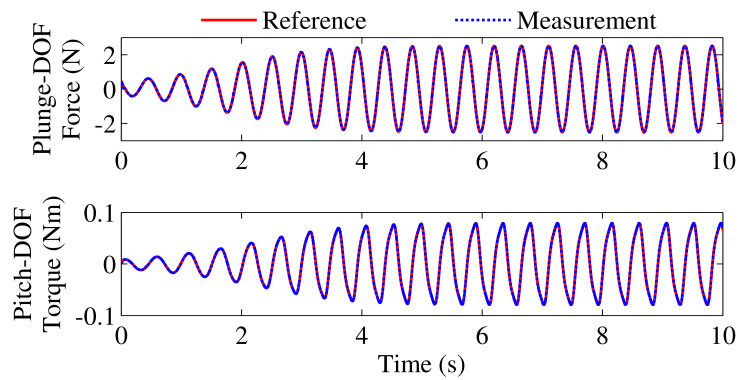

Fig. 11. VSDS force/torque under UIE-LQG control in Case 1 tests.
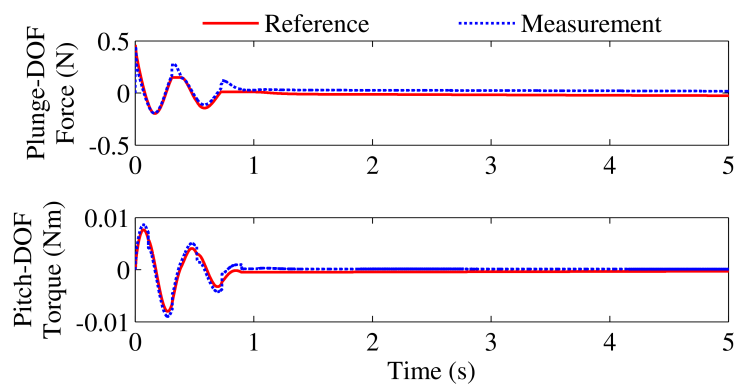

Fig. 12. VSDS force/torque under LQG control in Case 2 tests.
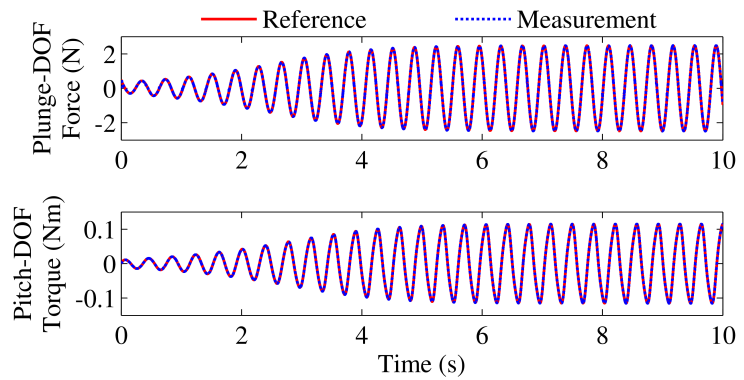

Fig. 13. VSDS force/torque under UIE-LQG control in Case 2 tests.

operation principle proposed effectively addresses dynamics coupling between each DOF without the need for sophisticated aeroelastic modeling. Resolution loss in velocity measurement is identified as a main problem associated with the first trial of non-reduction transmission on a VSDS and is solved by the proposed MESO. The proposed VSDS control system with UIE-LQG control enables robust force/torque tracking and significantly reduces system identification and calibration procedures in VSDS development, although with some phase lag introduced. Wind-tunnel experiments confirms that the new 2-DOF VSDS prototype can provide satisfactory simulation of structural stiffness and damping for AAT. Future work will focus on further reduction of the phase lag introduced by the control system.

\section{REFERENCES}

[1] M. G. Farmer, "A two-degree-of-freedom flutter mount system with low damping for testing rigid wings at different angles of attack," National Aeronautics and Space Administration, Report NASA TM 83302, Apr. 1982.

[2] G. D. Miller, "Active flexible wing (AFW) technology," Air Force Wright Aeronautical Laboratories, Report AFWAL-TR-87-3096, Feb. 1988.

[3] T. O'Neil and T. W. Strganac, "Aeroelastic response of a rigid wing supported by nonlinear springs," J. Aircr., vol. 35, no. 4, pp. 616-622, Jul-Aug 1998

[4] E. L. Burnett, J. A. Beranek, B. T. Holm-Hansen, C. J. Atkinson, and P. M. Flick, "Design and flight test of active flutter suppression on the X-56A multi-utility technology test-bed aircraft," Aeronaut. J., vol. 120, no. 1228, pp. 893-909, Jun. 2016.

[5] F. S. Hover, S. N. Miller, and M. S. Triantafyllou, "Vortex-induced vibration of marine cables: Experiments using force feedback," J. Fluids Struct., vol. 11, no. 3, pp. 307-326, Apr. 1997.

[6] F. S. Hover, A. H. Techet, and M. S. Triantafyllou, "Forces on oscillating uniform and tapered cylinders in crossflow," J. Fluid Mech., vol. 363, pp. 97-114, May 1998.

[7] J. F. Derakhshandeh, M. Arjomandi, B. S. Cazzolato, and B. Dally, "Harnessing hydro-kinetic energy from wake-induced vibration using virtual mass spring damper system," Ocean Eng., vol. 108, pp. 115128, Nov. 2015 .

[8] J. H. Lee, N. Xiros, and M. M. Bernitsas, "Virtual damper-spring system for VIV experiments and hydrokinetic energy conversion," Ocean Eng., vol. 38, no. 5-6, pp. 732-747, Apr. 2011.

[9] J. Lee and M. Bernitsas, "High-damping, high-reynolds VIV tests for energy harnessing using the VIVACE converter," Ocean Eng., vol. 38, no. 16, pp. 1697-1712, 2011.

[10] H. Sun, E. S. Kim, M. P. Bernitsas, and M. M. Bernitsas, "Virtual springdamping system for flow-induced motion experiments," J. Offshore Mech. Arct., vol. 137, no. 6, Dec. 2015.

[11] A. Kelly, Mobile Robotics: Mathematics, Models, and Methods. New York, NY: Cambridge University Press, 2013.

[12] R. D. Lorenz and K. W. Van Patten, "High-resolution velocity estimation for all-digital, AC servo drives," IEEE Trans. Ind. Appl., vol. 27, no. 4, pp. 701-705, Jul/Aug 1991

[13] R. Petrella, M. Tursini, L. Peretti, and M. Zigliotto, "Speed measurement algorithms for low-resolution incremental encoder equipped drives: A comparative analysis," in Proc. Int. Aegean Conf. Elect. Mach. Power Electron., Sep. 2007, pp. 780-787.

[14] T. Shi, Z. Wang, and C. Xia, "Speed measurement error suppression for PMSM control system using self-adaption Kalman observer," IEEE Trans. Ind. Electron., vol. 62, no. 5, pp. 2753-2763, May 2015.

[15] J. Han, "From PID to active disturbance rejection control," IEEE Trans. Ind. Electron., vol. 56, no. 3, pp. 900-906, Mar. 2009.

[16] G. Herbst, "Practical active disturbance rejection control: Bumpless transfer, rate limitation, and incremental algorithm," IEEE Trans. Ind. Electron., vol. 63, no. 3, pp. 1754-1762, Mar. 2016.

[17] Y. Yuan, P. Zhang, Z. Wang, L. Guo, and H. Yang, "Active disturbance rejection control for the ranger neutral buoyancy vehicle: A delta operator approach," IEEE Trans. Ind. Electron., vol. 64, no. 12, pp. 9410-9420, Dec. 2017.

[18] H. Yang, J. Sun, Y. Xia, and L. Zhao, "Position control for magnetic rodless cylinders with strong static friction," IEEE Trans. Ind. Electron., vol. 65, no. 7, pp. 5806-5815, Jul. 2018

[19] D. Tang, L. Chen, E. Hu, and Z. F. Tian, "A novel actuator controller: Delivering a practical solution to realization of active-truss-based morphing wings," IEEE Trans. Ind. Electron., vol. 63, no. 10, pp. 6226-6237, Oct. 2016 